\title{
Epicutaneous patch testing in drug hypersensitivity syndrome (DRESS)
}

\author{
Felicidade Santiago $^{1}$, Margarida Gonçalo ${ }^{1}$, Ricardo Vieira $^{1}$, Sónia Coelho $^{2}$ and Américo Figueiredo ${ }^{1}$ \\ ${ }^{1}$ Department of Dermatology, Coimbra University Hospital, Coimbra, and ${ }^{2}$ Department of Dermatology, \\ Hospital Infante D. Pedro, Aveiro, Portugal
}

Background: In some patterns of cutaneous adverse drug reactions, and depending on the culprit drug, patch testing has been helpful in confirming its cause. Its value in Drug Rash with Eosinophilia and Systemic Symptoms (DRESS) has not been established in a large cohort of patients.

Objective: The aim of the present study is to evaluate the safety and usefulness of patch testing in DRESS.

Patients/Methods: Between January 1998 and December 2008, we studied 56 patients with DRESS induced by antiepileptic agents in 33 patients (59\%), allopurinol in $19(34 \%)$ and sulfasalazine, cotrimoxazole, tenoxicam, and amoxicillin in 1 patient each $(7 \%)$.

Results: A positive patch test reaction was observed in 18 patients (32.1\%), of which 17 were with antiepileptics and 1 with tenoxicam. In the antiepileptic group, carbamazepine alone was responsible for 13 of 17 positive reactions $(76.5 \%)$. Patch tests with allopurinol and its metabolite were negative in all cases attributed to this drug.

Conclusions: In this study, patch testing was a safe and useful method in confirming the culprit drug in DRESS induced by antiepileptic drugs, whereas it had no value in DRESS induced by allopurinol. The pathogenesis of DRESS is not yet entirely clarified, but positive patch tests suggest a drug-dependent delayed hypersensitivity mechanism.

Key words: Allopurinol; antiepileptics; carbamazepine; DRESS; lamotrigine; patch testing. (C) John Wiley \& Sons A/S, 2010.

Conflicts of interest: None.

Accepted for publication 23 September 2009

Drug hypersensitivity syndrome-also known as 'Drug Rash with Eosinophilia and Systemic Symptoms' (DRESS) - is an uncommon, idiosyncratic adverse drug reaction with a delayed onset, a prolonged course, and a fatality rate of around $10-40 \%$. The number of causative drugs is expanding. The most frequent are aromatic antiepileptics such as carbamazepine, phenytoin and phenobarbital, dapsone, allopurinol, minocycline, and antiretroviral drugs like nevirapine and abacavir (1-4).

The pathogenesis of DRESS is complex and not fully understood: (i) genetic deficiency of detoxifying enzymes such as epoxide hydrolase, resulting in the accumulation of toxic drug metabolites (1); (ii) certain HLA haplotypes predispose to DRESS, like HLA-B*5701 for abacavir and HLA-B*5801 for allopurinol $(5,6)$; (iii) virus - drug interactions, as in patients where HHV-6 reactivates during DRESS (7); (iv) drug-induced immunosuppression followed by an inflammatory immune recovery syndrome is another possible explanation (8); and (v) drug-specific T-cells have been isolated from the blood and skin in DRESS induced by lamotrigine and carbamazepine, therefore suggesting a T-cell-mediated reaction (9-11). Recently, DRESS was classified under a delayed type IVb hypersensitivity reaction, where T-helper type 2 cells play a significant role (12-14), even though in abacavir 
hypersensitivity CD8+ T-cells seem to be preferentially involved (6).

To confirm the responsible agent in DRESS, rechallenge with the suspected drug, considered the 'gold standard' in other drug eruptions, is not advised due to the risk of a life-threatening reaction (15). Lymphocyte transformation/activation testing is cumbersome to perform, not standardized for most drugs, with a low sensitivity and specificity, and, therefore, it is mainly used in research centres $(12,16)$. It is also reported to be negative during the acute phase of DRESS (17).

Patch testing, performed in the study of drug eruptions, is not fully standardized, but it can be helpful in confirming the imputability of a drug in several patterns of cutaneous adverse drug reactions where delayed hypersensitivity mechanisms are involved. Positive patch tests occur more frequently in maculopapular exanthemata, acute generalized exanthematous pustulosis and fixed drug eruptions. In Stevens-Johnson syndrome and toxic epidermal necrolysis, patch testing is seldom positive (18). There are no large studies evaluating the utility of patch testing patients with DRESS. There are several controlled studies, with large numbers of patients, some with well-characterized patterns of drug eruptions and including cases of DRESS, but the results are usually evaluated as a whole $(15,19)$. Apart from the pattern of cutaneous adverse drug reactions, reactivity on patch testing is highly dependent on the culprit drug (15). Although the main drugs involved in DRESS have also been implicated in other cutaneous adverse drug reactions, it is important to evaluate if patch test reactivity is similar in this reaction pattern.

The aim of the present study was to evaluate the safety and usefulness of patch testing in this severe, potentially life-threatening adverse drug reaction, where rechallenge is contraindicated and intradermal testing is not recommended as a first line study.

\section{Patients and Methods}

\section{Patient selection and characterization}

From January 1998 to December 2008, in the Dermatology Department of Coimbra University Hospital, almost exclusively in the inpatient unit, 84 patients (50 females and 34 males with a mean age of 56.7 years) were diagnosed with DRESS. The diagnosis was based on the presence of a minimum of three criteria defined by Bocquet et al. (3) and on the scoring system proposed by RegiSCAR study group (20).

For the present study, and according to the French Pharmacovigilance criteria proposed by Moore et al. (21) and modified by Bégaud et al. (22), patients were included when one drug could be identified with an intrinsic imputability score of 3 (likely) or 4 (very likely), or, at least, one drug had a higher or much higher imputability score than the other drugs taken concomitantly by the patient.

Out of the 84 patients diagnosed in the department, only 56 patients (38 females and 18 males) with a mean age of $52.9 \pm 21.2$ years (range, 12-93) were available for performing patch testing. Some patients, due to their age, co-morbidities, multiple drug regimen or persistent immunosuppression for other diseases, were not included in the present study. According to the RegiSCAR criteria, 11 patients were possible cases (3 scored 2; 8 scored 3), 30 patients were probable cases (13 scored $4 ; 17$ scored 5$)$ and 15 patients were definite cases (8 scored 6; 4 scored 7; 3 scored 8). As we did not perform skin biopsies or autoimmunity studies in older patients, according to RegiSCAR criteria the DRESS scores were rather low in some patients.

Based on the clinical data and chronology, antiepileptic agents were the most frequently suspected triggering agents in 33 patients $(59 \%)$ followed by allopurinol in 19 (34\%). In three patients (one from carbamazepine and two from allopurinol) an accidental drug re-exposure with recurrence of the skin and systemic symptoms reinforced drug imputability. Other drugs (sulfasalazine, cotrimoxazole, tenoxicam, and amoxicillin) had a high clinical imputability score in four patients $(7 \%)$.

In the 56 patients, cutaneous manifestations of DRESS developed within $28.5 \pm 9.7$ days (range, 5-60) after drug administration and consisted on a generalized maculopapular rash involving more than $50 \%$ of the body surface in 51 patients $(91 \%)$ or erythroderma in $5(9 \%)$, and facial oedema in $39(69.6 \%)$. Systemic manifestations included pyrexia in 39 patients $(69.6 \%)$, lymphadenopathy in $23(41 \%)$ and liver involvement in 47 $(83.9 \%), 5$ of whom suffered temporary, severe liver failure. Increased serum creatinine and/or urea nitrogen was observed in 12 patients $(21.4 \%)$, and 4 patients $(7.1 \%)$ had pulmonary manifestations. Atypical circulating lymphocytes were found in 3 patients $(5.3 \%)$ and eosinophilia (>800 eosinophils/ $\mu \mathrm{l})$ in $30(53.6 \%), 12$ of them with more than 1500 eosinophils/ $\mu$ l. All patients recovered with systemic steroids and early withdrawal of the culprit drug, with a slowly progressive resolution of skin and systemic symptoms (mean 29.4 days). Aromatic antiepileptics were replaced by sodium valproate with a very good tolerance; in one case of carbamazepine-induced DRESS, administration of phenytoin 20 days 
later induced a reactivation of the skin and liver manifestations.

\section{Patch testing}

After informed patient consent, patch tests were performed 6 weeks to 6 months after complete healing of the adverse drug reaction, and at least 1 month after discontinuation of systemic corticosteroids, according to the proposed guidelines for performing patch tests in cutaneous adverse drug reactions (19).

Patch tests were applied for 2D on the upper back, using Finn ${ }^{\circledR}$ Chambers on Scanpor tape ${ }^{\circledR}$ (Epitest Ltd., Oy). Readings were performed at D2 and D3 or D4 according to the International Contact Dermatitis Research Group guidelines, with the advice of a referral reading at D7 in case of a late reaction. Only $1+$ or more intense reactions were considered.

All patients were patch tested with the European baseline series of contact allergens, with the culprit drug, with chemically or pharmacologically related drugs [series of antiepileptics, antibiotics, or nonsteroidal anti-inflammatory drugs (NSAIDs)] and with other drugs introduced in the previous weeks.

Patch tests were performed with the powder of the drug supplied by the pharmaceutical industry in a pure form $(>95 \%)$ and appropriately diluted in our laboratory or, more recently, with prepared drug allergens obtained from Chemotechnique ${ }^{\circledR}$ Diagnostics, Vellinge, Sweden. As we could not obtain the pure substance for Topamax ${ }^{\circledR}$ (Topiramate) and Salazopirine ${ }^{\circledR}$ (Sulfasalazine), we used the powder of the commercial drug for patch testing.

When antiepileptics were suspected, the 33 patients were tested with carbamazepine diluted in petrolatum (pet.) in several concentrations $(1 \%$, $5 \%, 10 \%$ pet. in all and $20 \%$ pet. additionally in 20 patients), lamotrigine (1\%, $10 \%$ pet.), phenytoin (5\%, $10 \%$ pet.), phenobarbital $(5 \%, 10 \%$ pet.), diazepam $(1 \%, 10 \%$ pet.), and, in 3 patients, also with Topamax $^{\circledR}$ powder at $30 \%$ in water and pet.

In the 19 cases where allopurinol was suspected, apart from allopurinol tested at 1-10-20\% pet. in all patients, in 9 patients patch tests were performed also with 8-oxypurinol (5-10\% pet.), and in 3 patients with allopurinol and oxypurinol at $10 \%$ in ethanol and water. In three patients, both allopurinol and oxypurinol in the highest concentrations in pet. were applied after tape stripping (23).

As control subjects for allopurinol and antiepileptics we have patch tested more than 50 patients with adverse drug reaction to other drugs and patients previously exposed to the drugs but with no reaction. In the case of Topamax ${ }^{\circledR}, 17$ controls with an

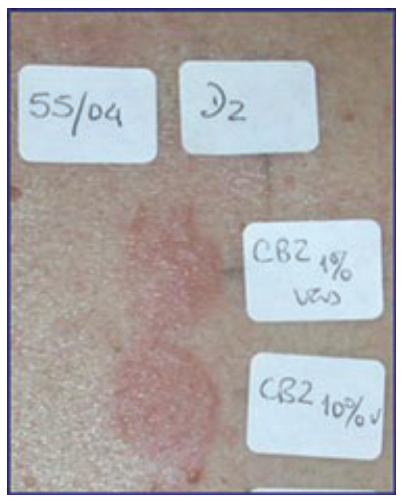

Fig. 1. Positive patch tests with carbamazepine at D2.

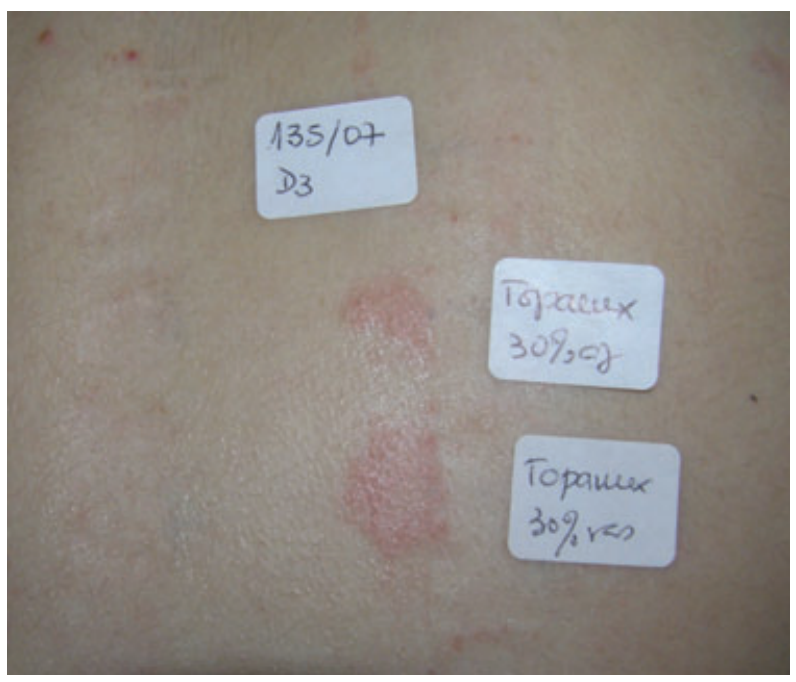

Fig. 2. Positive patch tests with Topamax ${ }^{\circledR}$ (topiramate).

adverse drug reaction to other antiepileptics were patch tested with the same preparation in pet.

\section{Results}

A positive patch test with the suspected drug (1+ or stronger) was observed in 18 of 56 patients $(32.1 \%)$, in 17 cases with antiepileptics and 1 with tenoxicam.

In the group of 33 patients with antiepilepticinduced DRESS, a positive result was observed in 17 cases (51.5\%). Carbamazepine alone was responsible for 13 of 17 positive patch tests (Fig. 1), followed by lamotrigine (2 cases), phenytoin (1 case) and Topamax ${ }^{\circledR}$ (1 case) (Fig. 2).

In overview, in the 18 patients where carbamazepine was suspected, 13 reacted positively (72.2\%), with $1+$ or $2+$ reactions (11 patients) and with $3+$ reactions (2 patients), apparently with no correlation of the intensity of reaction and the clinical score of the DRESS, although positive reactions 
Table 1. Patch tests results with antiepileptics and allopurinol

\begin{tabular}{|c|c|c|c|}
\hline Culprit drugs & Patients tested & Positive patch tests & $\%$ positive patch tests \\
\hline Antiepileptics & 33 & 17 & 51.5 \\
\hline Carbamazepine $(1 \%, 5 \%, 10 \%, 20 \%$ a pet.) & 18 & 13 & 72.2 \\
\hline Phenytoin $(5 \%, 10 \%$ pet.) & 7 & 1 & 14.3 \\
\hline Lamotrigine $(1 \%, 10 \%$ pet.) & 5 & 2 & 40 \\
\hline Phenobarbital (5\%, 10\% pet.) & 2 & 0 & 0 \\
\hline Topamax $^{\circledR}$ (topiramate, $30 \%$ in water and pet.) & 1 & 1 & 100 \\
\hline Allopurinol $(1 \%, 10 \%, 20 \% \text { pet. })^{\mathrm{b}}$ & 19 & 0 & 0 \\
\hline Oxypurinol $(5 \%, 10 \% \text { pet. })^{\mathrm{b}}$ & 9 & 0 & 0 \\
\hline
\end{tabular}

pet. - petrolatum.

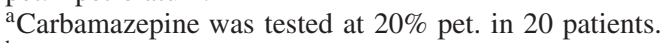

${ }^{\mathrm{b}}$ Allopurinol and oxypurinol were also tested at $10 \%$ in ethanol and water in three patients.

occurred in the more severe cases. Patients reacted to all the concentrations of carbamazepine tested, most of them with the more intense reaction at D2 or D3, and with no significant difference in reaction intensity between the concentration of $1 \%$ and $20 \%$. Therefore, by increasing the concentration from $1 \%$ to $20 \%$, no more reactive patients were detected. Relevance was confirmed by a positive accidental rechallenge in one patient.

Although patients were tested with all the antiepileptics, they reacted only to one drug, except in one case: a patient with a carbamazepine-induced DRESS that was reactivated with phenytoin reacted simultaneously to carbamazepine and phenytoin on patch testing.

None of the 19 patients patch tested with allopurinol nor the 9 tested with oxypurinol in several concentrations and vehicles developed a positive reaction (Table 1). In two of these patients additional lymphocyte activation tests performed with allopurinol and oxypurinol were negative. Nevertheless, 2 of these 19 patients had a positive accidental drug rechallenge: one had a maculopapular exanthema with no systemic symptoms on the first episode, and, 2 years later, a DRESS with allopurinol readministration.

An isolated positive reaction was observed with tenoxicam, but with no reaction to piroxicam, meloxicam, or other NSAIDs.

There were no positive patch tests in controls and no adverse reaction, namely no reactivation of skin lesions or systemic symptoms during or after patch testing.

There was no particular reactivity within the European baseline series: 18 positive reactions $(32.1 \%)$ were observed, 6 patients had also positive patch tests to the suspected drugs, and 12 had isolated reactivity within the baseline series. The main allergens within the baseline series were nickel sulfate (55.5\%), cobalt chloride (11.1\%), and $p$-phenylenediamine (11.1\%).

\section{Discussion}

The usefulness of skin testing in the study of cutaneous adverse drug reactions, including patch, prick, and intradermal testing with immediate and delayed readings, varies significantly according to the drug tested and the clinical features of the drug eruption, and depends highly on patient selection $(15,24)$. Considering only patch testing, reactivity occurs in less than $50 \%$ of patients (19), varying from $10.8 \%$ among 826 patients studied (25) to $43.9 \%$ in 66 patients (23).

It is not possible to perform patch testing during the acute reaction; often there is no clear skin, and iatrogenic immunosuppression might reduce skin reactivity. Therefore, patch testing has to be delayed at least 4-6 weeks after resolution of the cutaneous adverse drug reaction.

As shown in our series, patch testing is generally a safe procedure, including in severe adverse drug reactions, like DRESS. Even when a drug is tested at higher concentrations (20\% pet.), the risk of developing a severe reaction is unlikely to occur. There is only one case published where patch testing induced an exfoliative dermatitis; however, it was performed using crushed tablets in pet. where final drug concentration could not be adequately controlled (26).

In several studies patch testing of patients with DRESS have been included, but this pattern of adverse drug reaction was not usually separated from other maculopapular exanthemata or exfoliative dermatitis, and, therefore, results on the value of patch testing in DRESS have not been clearly defined. Although drug-specific T-cells have been isolated from the blood and skin in patients with DRESS, the pathogenesis of this adverse drug reaction seems to be much more complex with acute viral reactivation and eventual viral - drug interactions, and, therefore, patch tests performed after the resolution of the adverse drug reaction might be unable to reproduce drug skin hypersensitivity. 
In this study, $32.1 \%$ of patients with DRESS had a positive patch test, which is in the normal range of reactivity for other delayed cutaneous adverse drug reactions $(23,25)$. Also, as in other drug reaction patterns, the results of patch testing in DRESS are highly dependent on the culprit drug $(15,24)$. This is exemplified by divergent results in the two major groups: reactivity of $51.5 \%$ in the antiepileptic group and no reactivity at all in the allopurinol group.

In the antiepileptic group, patch test positivity was high, particularly for carbamazepine (72.2\%). Previous studies have shown the usefulness of patch testing in the diagnosis of hypersensitivity to antiepileptics, particularly for carbamazepine, with positive reactions in $70-100 \%$ of the cases studied $(1,12,19,27-30)$. Nevertheless, even for carbamazepine, patch test reactivity depends on the pattern of the cutaneous adverse drug reactions (30). We have shown that in patients with DRESS reactivity to carbamazepine is high (72.2\%), and, therefore, patch testing is a very useful complementary test to confirm its participation in this cutaneous adverse drug reaction.

Although the recommended concentration for testing most drugs in cutaneous adverse drug reactions is $10 \%$ pet. (19), in the case of carbamazepine we have previously tried to increase the concentration up to $20 \%$ to evaluate if we could detect more reactive patients. We have previously shown, in different types of cutaneous adverse drug reactions from carbamazepine, that patch testing yielded the same results with concentrations of carbamazepine ranging from $1 \%$ to $20 \%$ (31). In 1975, Houwerzijl et al. reported six out of seven patients with a severe rash and general symptoms with positive patch tests at all the concentrations of carbamazepine tested $(10 \%, 20 \%, 40 \%$ pet.) (27), and Silva et al. described three patients with exfoliative erythroderma with strongly positive patch test reactions to $1 \%, 2 \%$, and $10 \%$ carbamazepine pet. (28). Some authors have described positive reactions with small drug concentrations, but no reactions to carbamazepine were elicited at concentrations lower than $0.1 \%(28-30)$. The present study, with all patients reacting to carbamazepine at $1 \%$ and $20 \%$ pet., with no significant difference in the intensity of the reaction, suggests that a low concentration (1\% pet.) is also valuable for using in DRESS.

Positive patch tests in phenytoin-induced DRESS are less frequent than for carbamazepine, and in our study only one patient out seven had a positive patch test to phenytoin at $5 \%$ and $10 \%$ pet., a result similar to previous reports $(32,33)$. There are also only some published reports with positive patch tests to lamotrigine $(32,34)$. To our knowledge the positive patch test reaction to topiramate is the second described in the literature (35).
In cutaneous adverse drug reactions, crossreactivity among aromatic antiepileptics occurs in about $80 \%$ of the individuals $(1,12)$, but this cross-reactivity was not evidenced in patch testing. Only one patient, with DRESS induced by carbamazepine that worsened when phenytoin was introduced on day 20, had a positive patch test to both drugs. Some authors have suggested that the supposed cross-reactions might not actually be due to a chemical or antigenic similitude between them, but rather to the fact that a second anticonvulsant is administered during the immunologic depression occurring during a first anticonvulsant-related DRESS (36).

Allopurinol, a xanthine oxidase inhibitor widely used to control plasma uric acid levels, is one of the drugs frequently reported to cause DRESS (4). When administered orally it is rapidly metabolized to its oxidative metabolite 8-oxypurinol, which is considered to be responsible for most of the actions of allopurinol. In the 19 patients studied, patch tests with allopurinol and its metabolite were always negative, as in our previous study involving 12 patients with several types of cutaneous adverse drug reactions to allopurinol, including 7 with DRESS (37) Even though the molecular weight (136.11) of allopurinol is small we performed tape stripping before applying the patches, but it did not enhance the reactivity. The negativity of these patch tests cannot exclude responsibility of allopurinol, as two of these patients, who were accidentally rechallenged orally, developed hypersensitivity. There are few reports on patch testing with allopurinol, and we found only one reference to a positive patch test to a commercial formulation of allopurinol tested at $20 \%$ or $30 \%$ pet. among 10 patients studied (25), but with no reference to the type of cutaneous adverse drug reaction. There is no definite explanation for the high number of false-negative results, but theoretically there may be several causes: (i) the final responsible agent is another drug metabolite that is not formed in the skin during patch testing; (ii) there is no immune mechanism involved; (iii) concomitant factors that are responsible in inducing transient oral drug intolerance, such as viral infection, are not present at the time of testing; and (iv) wrong choice of vehicle, drug concentration, and exposure time $(23,37)$.

Like patch testing, lymphocyte stimulation testing in allopurinol-induced cutaneous adverse drug reaction has also been inconclusive. In one single report with three patients, Hamanaka et al. (38) had significant lymphoproliferative reactions to oxypurinol, not to allopurinol, which led them to consider that DRESS was due to oxypurinol. However, our studies do not seem to corroborate this possibility. In order to better explore the non-reactors, intradermal 
testing could be tried in a hospital setting, although, as allopurinol is not highly soluble in water, it might be difficult to have a sterilized homogeneous preparation in saline for intradermal testing. Alternatively we could try administration of the drug by iontophoresis.

As a conclusion, we consider patch testing a safe procedure to study patients with DRESS. Although it is useful in patients with antiepileptic druginduced DRESS, where the proportion of relevant and specific positive patch tests is high, when allopurinol is the suspected drug, patch testing has no value.

Apart from its usefulness in confirming the culprit drug in DRESS, patch testing also contributes to the understanding of the pathomechanisms involved. A specific positive patch test reaction reinforces the involvement of drug-dependent delayed hypersensitivity mechanisms in this complex cutaneous adverse drug reaction, and can be useful to collect drug-specific T-cells for further studies.

\section{References}

1. Shear N H, Spielberg S P. Anticonvulsant hypersensitivity syndrome. In vitro assessment of risk. J Clin Invest 1988: 82: $1826-1832$

2. Schlienger R G, Shear N H. Antiepileptic drug hypersensitivity syndrome. Epilepsia 1998: 39: S3-S7.

3. Bocquet H, Bagot M, Roujeau J C. Drug-induced pseudolymphoma and drug hypersensitivity syndrome (Drug Rash with Eosinophilia and Systemic Symptoms: DRESS). Semin Cutan Med Surg 1996: 15: 250-257.

4. Roujeau J C, Stern R S. Severe adverse cutaneous reactions to drugs. N Engl J Med 1994: 331: 1272-1285.

5. Chung W H, Hung S I, Chen Y T. Human leukocyte antigens and drug hypersensitivity. Curr Opin Allergy Clin Immunol 2007: 7: 317-323.

6. Phillips E J, Mallal S A. HLA and drug-induced toxicity. Curr Opin Mol Ther 2009: 11: 231-242.

7. Descamps V, Valance A, Edlinger C, Fillet A M, Grossin M, Lebrun-Vignes B, Belaich S, Crickx B. Association of human herpesvirus 6 infection with drug reaction with eosinophilia and systemic symptoms. Arch Dermatol 2001: 137: 301-304.

8. Kano Y, Inaoka M, Shiohara T. Association between anticonvulsant hypersensitivity syndrome and human herpesvirus 6 reactivation and hypogammaglobulinemia. Arch Dermatol 2004: 140: 183-188.

9. Knowles S R, Uetrecht J, Shear N H. Idiosyncratic drug reactions: the reactive metabolite syndromes. Lancet 2000: 356: 1587-1591.

10. Naisbitt DJ, Farrell J, Wong G et al. Characterization of drugspecific $\mathrm{T}$ cells in lamotrigine hypersensitivity. J Allergy Clin Immunol 2003: 111: 1393-1403.

11. Naisbitt D J, Britschgi M, Wong G et al. Hypersensitivity reactions to carbamazepine: characterization of the specificity, phenotype, and cytokine profile of drug-specific T cell clones. Mol Pharmacol 2003: 63: 732-741.

12. Knowles S R, Shapiro L E, Shear N H. Anticonvulsant hypersensitivity syndrome: incidence, prevention and management. Drug Saf 1999: 21: 489-501.

13. Lerch M, Pichler W J. The immunological and clinical spectrum of delayed drug-induced exanthems. Curr Opin Allergy Clin Immunol 2004: 4: 411-419.
14. Pichler W, Yawalkar N, Schmid S, Helbling A. Pathogenesis of drug-induced exanthems. Allergy 2002: 57: 884-893.

15. Barbaud A, Reichert-Penetrat $S$, Tréchot $P$ et al. The use of skin testing in the investigation of cutaneous adverse drug reactions. Br J Dermatol 1998: 139: 49-58.

16. Spielberg $S$. In vitro analysis of idiosyncratic drug reactions. Clin Biochem 1986: 19: 142-144.

17. Kano Y, Hirahara K, Mitsuyama Y et al. Utility of the lymphocyte transformation test in the diagnosis of drug sensitivity: dependence on its timing and the type of drug eruption. Allergy 2007: 62: 1439-1444.

18. Wolkenstein P, Chosidow O, Fléchet M L, Robbiola O, Paul M, Dumé L, Revuz J, Roujeau J C. Patch testing in severe cutaneous adverse drug reactions, including Stevens-Johnson syndrome and toxic epidermal necrolysis. Contact Dermatitis 1996: 35: 234-236.

19. Barbaud A, Gonçalo M, Bruynzeel D, Bircher A. European Society of Contact Dermatitis. Guidelines for performing skin tests with drugs in the investigation of cutaneous adverse drug reactions. Contact Dermatitis 2001: 45: 321-328.

20. Kardaun S H, Sidoroff A, Valeyrie-Allanore L, Halevy S, Davidovici B B, Mockenhaupt M, Roujeau J C. Variability in the clinical pattern of cutaneous side-effects of drugs with systemic symptoms: does a DRESS syndrome really exist? Br J Dermatol 2007: 156: 609-611.

21. Moore N, Biour M, Paux G, Loupi E, Begaud B, Boismare F, Royer R J. Adverse drug reaction monitoring: doing it the French way. Lancet 1985: 2: 1056-1058.

22. Bégaud B, Evreux J C, Jouglard J, Lagier G. Imputation of the unexpected or toxic effects of drugs. Actualization of the method used in France. Therapie 1985: 40: $111-118$.

23. Gonçalo M, Fernandes B, Oliveira H, Figueiredo A. Epicutaneous patch testing in drug eruptions. Contact Dermatitis 2000: 42: 52.

24. Barbaud A. Drug patch testing in systemic cutaneous drug allergy. Toxicology 2005: 209: 209-216.

25. Lammintausta K, Kortekangas-Savolainen O. The usefulness of skin tests to prove drug hypersensitivity. $\mathrm{Br} J$ Dermatol 2005: 152: 968-974.

26. Vaillant L, Camenen I, Lorette G. Patch testing with carbamazepine: reinduction of an exfoliative dermatitis. Arch Dermatol 1989: 125: 299.

27. Houwerzijl J, DeGast G, Nater J. Patch tests in drug eruptions. Contact Dermatitis 1975: 1: 180-192.

28. Silva R, Machado A, Brandão M, Gonçalo S. Patch test diagnosis in carbamazepine erythroderma. Contact Dermatitis 1986: 15: 254-255.

29. Alanko K. Patch testing in cutaneous reactions caused by carbamazepine. Contact Dermatitis 1993: 29: 254-257.

30. Puig L, Nadal C, Fernández-Figueras M T, Alomar A. Carbamazepine induced drug rashes: diagnostic value of patch tests depends on clinico-pathologic presentation. Contact Dermatitis 1996: 34: 435-437.

31. Gonçalo M, Coelho S, Figueiredo A. Ascertaining patch test concentration in cutaneous adverse drug reactions to aminopenicillins and carbamazepine. J Invest Dermatol 2006: 126: S3-S67.

32. Galindo P A, Borja J, Gómez E et al. Anticonvulsant drug hypersensitivity. J Invest Allergol Clin Immunol 2002: 12: 299-304.

33. Lee A Y, Kim M J, Chey W Y, Choi J, Kim B G. Genetic polymorphism of cytochrome P450 2C9 in diphenylhydantoininduced cutaneous adverse drug reactions. Eur J Clin Pharmacol 2004: 60: 155-159.

34. Monzón S, Garcés M M, Reichelt C, Lezaun A, Colás C. Positive patch test in hypersensitivity to lamotrigine. Contact Dermatitis 2002: 47: 361 .

35. Schiavino D, Nucera E, Buonomo A et al. A case of type IV hypersensitivity to topiramate and carbamazepine. Contact Dermatitis 2005: 52: 161-162. 
36. Klassen B D, Sadler R M. Induction of hypersensitivity to a previously tolerated antiepileptic drug by a second antiepileptic drug. Epilepsia 2001: 42: 433-435.

37. Vieira R, Gonçalo M, Figueiredo A. Testes epicutâneos ao alopurinol e oxipurinol em doentes com toxidermias ao alopurinol. Trab Soc Port Dermatol Venereol 2004: 62: 247-253.

38. Hamanaka H, Mizutani H, Nouchi N, Shimizu Y, Shimizu M. Allopurinol hypersensitivity syndrome: hypersensitivity to oxypurinol but not allopurinol. Clin Exp Dermatol 1998: 23: $32-34$.
Address:

Felicidade Santiago

Department of Dermatology

Coimbra University Hospital

Praceta Mota Pinto

3000-075 Coimbra

Portugal

Tel: 00351239400420

Fax: 00351239400490

e-mail: felicidadesantiago@hotmail.com 